\title{
Targeting of cancer-associated fibroblasts enhances the efficacy of cancer chemotherapy by regulating the tumor microenvironment
}

\author{
MINMIN LI ${ }^{1 *}$, MEI LI $^{1 *}$, TAO YIN ${ }^{1 *}$, HUASHAN SHI ${ }^{1}$, YUAN WEN $^{2}$, BINGLAN ZHANG $^{1}$, \\ MEIHUA CHEN ${ }^{1}$, GUANGCHAO XU ${ }^{2}$, KEXIN REN $^{1}$ and YUQUAN WEI ${ }^{1,3}$ \\ ${ }^{1}$ Department of Medical Oncology, Cancer Center, State Key Laboratory of Biotherapy, West China Hospital; \\ ${ }^{2}$ State Key Laboratory of Biotherapy, West China Hospital and School of Life Science, \\ Sichuan University, Chengdu, Sichuan 610041, P.R. China
}

Received December 30, 2014; Accepted September 24, 2015

DOI: $10.3892 / \mathrm{mmr} .2016 .4868$

\begin{abstract}
Cancer-associated fibroblasts (CAFs), key components of the tumor stroma, can regulate tumorigenesis by altering the tumor microenvironment in variety of ways to promote angiogenesis, recruit inflammatory immune cells and remodel the extracellular matrix. Using a murine xenograft model of colon carcinoma, the present study observed that oxaliplatin increased the accumulation of CAFs and stimulated the production of cytokines associated with CAFs. When oxaliplatin was combined with the small-molecule dipeptidyl peptidase inhibitor PT-100, which inhibits CAFs by targeting fibroblast activation protein (FAP), the accumulation of CAFs was markedly reduced, xenograft tumor growth was significantly suppressed and the survival of the mice increased, compared to those of mice treated with oxaliplatin or PT-100 alone. Furthermore, the xenograft tumor tissues of mice treated with oxaliplatin and PT-100 contained lower numbers of tumor-associated macrophages and dendritic cells, expressed lower levels of cytokines associated with CAFs and had a lower density of CD31+ endothelial cells. The present study demonstrated that pharmacological inhibition of CAFs improved the response to chemotherapy, reduced the recruitment of immune tumor-promoting cells and inhibited angiogenesis. Combining chemotherapy with agents which target CAFs may represent a novel strategy for improving the efficacy of chemotherapy and reducing chemoresistance.
\end{abstract}

Correspondence to: Professor Yuquan Wei, Department of Medical Oncology, Cancer Center, State Key Laboratory of Biotherapy, West China Hospital, Sichuan University, 17 Renmin South Road, Chengdu, Sichuan 610041, P.R. China

E-mail: yqwei@scu.edu.cn

*Contributed equally

Key words: cancer-associated fibroblasts, fibroblast activation protein, tumor microenvironment, PT-100, chemotherapy

\section{Introduction}

Tumors are complex structures composed of malignant cancer cells surrounded by the tumor stroma. The cells and components of the tumor stroma have received increasing attention due their roles in tumor development, invasion and metastasis (1), and in the response to cancer therapy $(2,3)$. The tumor stroma contains various cell types, including myeloid cell sub-populations, inflammatory cells, immunocytes, endothelial cells, epithelial cells and fibroblasts $(4,5)$, which communicate between themselves and also directly with the cancer cells through cell-cell contacts and indirectly through paracrine/exocrine signaling, release of proteases and modulation of the extracellular matrix (ECM). Together, the stromal cells constitute the complex tumor microenvironment, which has a key role in tumor development $(1,6)$. On this basis, identification of stromal targets for cancer therapeutics is of great interest, and such strategies may complement therapies directed against cancer cells. Among the potential targets in the stroma, cancer-associated fibroblasts (CAFs) have received intensive interest.

It is thought that the fibroblasts in the tumor stroma acquire a modified phenotype, which can be utilized for distinguishing them from normal tissue fibroblasts. Such 'activated' fibroblasts have been termed peritumoral fibroblasts, reactive stromal fibroblasts, myofibroblasts or CAFs (7). The precise origin of CAFs remains to be elucidated; however, a previous study has identified two potential pathways: Resident fibroblasts may be converted into CAFs through stimulation by cytokines, including transforming growth factor-beta (TGF- $\beta$ ) and stromal cell-derived factor-1 (SDF-1) (8); furthermore epithelial or endothelial cells may transform into CAFs via epithelial-mesenchymal transition (EMT) and endothelial-to-mesenchymal transition (EndMT), which are also mediated by cytokines, including fibroblast growth factor (FGF), osteopontin (9), TGF- $\beta$ and SDF-1 $(10,11)$.

Numerous studies have provided evidence for the cancer-promoting role of CAFs. In contrast to resting fibroblasts, CAFs are characterized by an increased rate of proliferation and differential expression of ECM components and growth factors, including TGF- $\beta$, vascular endothelial growth factor (VEGF), platelet-derived growth factor (PDGF) and fibroblast growth factor 2 (FGF-2) $(12,13)$. These cytokines and growth factors have all been show to have important roles in synchronizing 
key events which continuously occur in the tumor microenvironment. For instance, TGF- $\beta$ promotes the infiltration of inflammatory/immune cells and CAFs into the tumor microenvironment, directly leading to changes in tumor cells (14). CAFs orchestrate tumor-promoting inflammation in a nuclear factor (NF) $-\kappa B$ signaling-dependent manner (15). VEGF induces microvascular permeability, leading to the extravasation of plasma proteins such as fibrin, which subsequently attracts CAFs, inflammatory immune cells and endothelial cells, leading to tumor angiogenesis (16). While CAFs and inflammatory cells are the principal sources of host-derived VEGF (16), PDGF and FGF-2 also have significant roles in angiogenesis. Therefore, CAFs are key factors that can promote tumor growth by inducing angiogenesis, recruiting inflammatory/immune cells and remodeling the ECM.

Compared to normal fibroblasts, extensive changes in the expression of genes that encode certain extracellular matrix proteins and proteases have been observed in CAFs in numerous types of carcinoma (17-19); among these, fibroblast activation protein (FAP), a type II membrane-bound serine protease, has recently gained attention. FAP has been shown to possess dipeptidyl peptidase- (20) and collagenase-like (21) activity in vitro, and has been implicated in ECM remodeling. However, the in vivo substrates of FAP remain to be identified. Due to its tightly regulated pattern of expression in the stroma of malignant solid tumors $(22,23)$, FAP has been classified as a candidate protein for targeting CAFs. The present study hypothesized that FAP inhibition may be useful for cancer therapy. FAP belongs to the post-proline dipeptidyl aminopeptidase family and has the highest similarity to dipeptidyl peptidase IV (DPPIV/CD26) (24). The catalytic sites of CD26/DPP-IV and FAP contain the characteristic catalytic triad of Ser ${ }^{630 / 624}$, $\mathrm{Asp}^{708 / 702}$ His $^{740 / 734}$ (the residues are numbered according to human CD26/DPP-IV and FAP, respectively), and the active serine is situated in a nucleophilic elbow motif within the sequence Gly-Trp-Ser-Tyr-Gly (13-15) $(25,26)$. The aminoboronic dipeptide and Val-boro-Pro (PT-100; Fig. 1) appear to be interesting drug candidates for the post-proline dipeptidyl aminopeptidase family. PT-100 competitively inhibits the DPP activity of FAP and CD26/DPP-IV, and forms a high-affinity interaction with the catalytic sites due to the formation of a complex between Ser ${ }^{630 / 624}$ and the boron atom of PT-100 (27).

Oxaliplatin is recognized as one of the standard drugs for chemotherapy of colorectal cancer in clinical practice; however, there is a certain risk of drug resistance and tumor recurrence in patients treated with oxaliplatin $(28,29)$, and a previous study demonstrated that these events may be associated with changes in CAFs (30). In order to further provide insight into the mechanisms by which CAFs contribute to tumor progression and resistance to chemotherapy, the present study investigated the combined effects of oxaliplatin and PT-100 in the treatment of CT26 colorectal cancer cell-derived tumors in a murine xenograft model, and observed the combined effects of oxaliplatin and PT-100 on the tumor microenvironment.

\section{Materials and methods}

Animals and cell lines. A total of 40 female BALB/c mice (6-8 weeks old and weighing $20 \mathrm{~g}$ ) were purchased from Beijing HFK Bioscience Co. Ltd., (Beijing, China) and maintained in

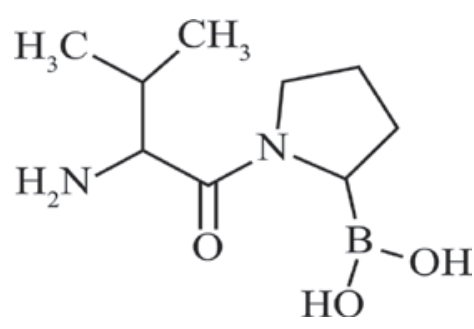

Figure 1. Chemical structure of PT-100.

a under a specific pathogen-free environment at the State Key Laboratory of Biotherapy (Chengdu, China) with controlled temperature $\left(20-26^{\circ} \mathrm{C}\right)$, humidity $(40-70 \%)$ and a 12-h light/dark cycle.

All animal experiments were approved by the Institutional Animal Care and Use Committee of Sichuan University (Chengdu, China). CT26, a BALB/c-derived murine colon carcinoma cell line, was purchased from the American Type Culture Collection (Manassas, VA, USA). CT26 cells were cultured in RPMI-1640 supplemented with $10 \%$ fetal bovine serum (Gibco; Thermo Fisher Scientific, Inc., Waltham, MA, USA) in a humidified atmosphere containing $5 \% \mathrm{CO}_{2}$ at $37^{\circ} \mathrm{C}$.

PT-100. PT-100 was purchased from Shanghai Speed Chemical Co. Ltd., (Shanghai, China). The purity was $>97 \%$. The molecular weight of PT-100 is $246.1 \mathrm{~g} / \mathrm{mol}$ and its structure is shown in Fig. 1.

Murine tumor xenograft model. The flanks of the mice were shaved and subcutaneously inoculated with $1 \times 10^{6}$ CT26 cells. The tumor-inoculated mice were divided into four groups: The saline vehicle group; the oxaliplatin group, which was treated with $5 \mathrm{mg} / \mathrm{kg}$ oxaliplatin (Jiangsu Hengrui Medicine Co., Ltd., Lianyungang, China) three times a week; the PT-100 group, which was treated with $20 \mu \mathrm{g}$ PT-100 per mouse daily; and a combined oxaliplatin and PT-100 group. All drugs were dissolved in saline and administered by intraperitoneal injection. The day of tumor inoculation was defined as day 0 , the treatment began on day 8 and animals were treated for 14 days. Tumor growth was monitored every 3 days by measurement of the length (L) and the width (W) of the xenograft tumors using Vernier calipers (Shanghai Taihai Measuring Tools Co., Ltd., Shanghai, China). The tumor volume was calculated using the following formula: Volume $\left(\mathrm{cm}^{3}\right)=\mathrm{W}^{2} \mathrm{x} 0.5 \mathrm{~L}$. The xenograft experiment was performed twice with 5 mice per group in each experiment.

Tissue preparation, histology and immunostaining. At the appropriate time-points, for paraffin embedding, the tumors were excised and post-fixed in $4 \%$ paraformaldehyde overnight. For cryopreservation, the tumors were excised and stored in optimum cutting temperature compound (OCT; Leica Microsystems $\mathrm{GmbH}$, Wetzlar, Germany) at $-20^{\circ} \mathrm{C}$.

FAP expression was assessed by immunohistochemistry in the CT26 colon cancer cell-derived xenograft tumors from female BALB/c mice. Tissues were cut into $5-\mu \mathrm{m}$ sections, incubated with $3 \% \mathrm{H}_{2} \mathrm{O}_{2}$ at $4^{\circ} \mathrm{C}$ for $20 \mathrm{~min}$, washed using phosphate-buffered saline (PBS) containing $3.74 \mathrm{~g} 12 \mathrm{H}_{2} \mathrm{O}_{2} \mathrm{Na}_{2} \mathrm{HPO}_{4}$, 
$0.44 \mathrm{~g} \mathrm{NaH}_{2} \mathrm{PO}_{4} .2 \mathrm{H} 2 \mathrm{O}$ and $7.2 \mathrm{~g} \mathrm{NaCl}$ in 11 distilled water $(\mathrm{PH}$ 7.4) and incubated with serum at $37^{\circ} \mathrm{C}$ for $20 \mathrm{~min}$. Subsequently, the sections were incubated with the following primary antibodies at $4^{\circ} \mathrm{C}$ overnight: Polyclonal anti-FAP (1:300; ab28244) $(v / v)$, monoclonal anti-vimentin $(1: 500 ;$ ab92547) $(v / v)$ and monoclonal anti-CD31 (1:50; ab7388; all Abcam, Cambridge, MA, USA) $(v / v)$. Following washing with PBS, the sections were incubated with a biotin-streptavidin-horseradish peroxidase (HRP) detection system (SP-9000; OriGene Technologies, Inc., Beijing, China) at $37^{\circ} \mathrm{C}$ for $1 \mathrm{~h}$, washed with PBS and subsequently incubated with 3,3'-diaminobenzidine (DAB) for 2 min and washed using PBS. The HRP-conjugated secondary antibody and the DAB were included in the detection system (SP-9000; ZSGB-Bio Origene Co, Ltd., Beijing, China), which was not diluted and was used according to the manufacturer's instructions. To assess the levels of apoptosis in CT26 tumors, in situ terminal deoxynucleotidyl transferase-mediated deoxy-UTP nick end labeling (TUNEL) staining was performed on the tumor sections using the the Deadend ${ }^{\mathrm{TM}}$ Fluorometric TUNEL system (G3250; Promega, Madison, WI, USA) according to the manufacturer's instructions.

For histological analysis, the paraffin-embedded tumor tissues were de-paraffinized in xylene, re-hydrated in 100, 95, 85 and then $75 \%$ ethanol, immersed in PBS (pH 7.4) and stained with hematoxylin (H3136-25G) and eosin (E4009-5G; both Sigma-Aldrich, St. Louis, MO, USA), according to the manufacturer's instructions. A Leica DM 2500 microscope was used for visualization (Leica Microsystems $\mathrm{GmbH}$ ).

Western blot analysis. On day 22, the tumors were excised and proteins were extracted using radioimmunoprecipitation assay buffer (Beyotime Institute of Biotechnology, Inc., Haimen, China) supplemented with protease inhibitor cocktail (100:1; Sigma-Aldrich). Tumor tissues were homogenized prior to western blot and PCR analysis in liquid nitrogen using a mortar and pestle. Total protein concentrations in the supernatant were determined via the Bicinchoninic Acid assay (P0013B; Beyotime Institute of Biotechnology, Inc.). A total of $30 \mu \mathrm{g}$ protein was loaded per lane for western blot analysis. Protein samples were separated by $10 \%$ SDS-PAGE and transferred onto polyvinylidene difluoride membranes (EMD Millipore, Billerica, MA, USA). After blocking with 5\% skimmed milk in TBS containing $0.1 \%$ Tween 20 (TBST) for $2 \mathrm{~h}$ at $37^{\circ} \mathrm{C}$, the membranes were incubated with anti-FAP $(1: 500 ; 28244$; Abcam) or anti- $\beta$-actin (1:1,000; sc-130657; Santa Cruz Biotechnology, Inc., Dallas, TX, USA) primary polyclonal antibodies at $4^{\circ} \mathrm{C}$ overnight. After washing four times with TBST for $10 \mathrm{~min}$, the membranes were incubated with the goat anti-rabbit HRP-conjugated secondary antibody (1:5,000; sc-2054; Santa Cruz Biotechnology, Inc.) for $1 \mathrm{~h}$ at $37^{\circ} \mathrm{C}$. After washing with TBST as described above, the bands were visualized using enhanced chemiluminescence reagents (WBKLS0100 EMD Millipore).

Flow cytometry. On day 22, the tumors were excised, dissected into small pieces using a scalpel, digested in collagenase digestion buffer (1\% collagenase in RPMI-1640; Gibco; Thermo Fisher Scientific, Inc.) for $2 \mathrm{~h}$ at $37^{\circ} \mathrm{C}$ with agitation and the cells in the resulting suspension were counted. Cells were passed through a $70 \mu \mathrm{m}$ cell strainer (BD Biosciences, Franklin Lakes, NJ, USA) following disaggregation A total of $1 \times 10^{6}$ cells in
$100 \mu \mathrm{l}$ were stained. The cells were labeled using rat anti-mouse CD11b-allophycocyanine (1:50; cat no. 553312; BD Biosciences), hamster anti-mouse CD11c-fluorescein isothiocyanate (1:100; cat no. 553801; BD Biosciences), or rat anti-mouse F4/80-A488 (1:50; cat no. MCA497A488; Serotec, Bio-Rad Laboratories, Inc., Hercules, CA, USA) antibodies on ice for $30 \mathrm{~min}$, followed by analysis by flow cytometry using FACSCalibur or LSR II flow cytometers (BD Biosciences); data were analyzed using CellQuest 6.0 software (BD Biosciences).

Reverse-transcription quantitative polymerase chain reaction $(R T-q P C R)$ analysis. Total RNA was extracted from the xenograft tumors using the AxyPrep ${ }^{\mathrm{TM}}$ Multisource Total RNA Miniprep kit (Axygen Biosciences, Union City, CA, USA) in accordance with the manufacturer's instructions. The PrimeScript $^{\mathrm{TM}}$ RT reagent kit with gDNA Eraser (Takara Bio Inc., Otsu, Japan) was used to synthesize cDNA, and RT-qPCR analysis was performed using SsoAdvanced ${ }^{\mathrm{TM}}$ SYBR Green Supermix (Bio-Rad Laboratories, Inc.) following the manufacturer's instructions. Transcript expression was determined relative to $G A P D H$. The primers used were purchased from BGI-Shenzhen (Shenzhen, China) and were as follows: GAPDH, 5'-ACCCAGAAGACTGTGGATGG-3' (forward) and 5'-TCT AGACGGCAGGTCAGGTC-3' (reverse); $T G F-\beta, 5^{\prime}$-AAGTGG GTCCATGAACCTAA-3' (forward) and 5'-GCTACATTTACA AGAC66CAC-3' (reverse); $F G F-2,5$ '-GGCTGCTGGCTTCTA AGTGT-3' (forward) and 5'-CCGTTTTGGATCCGAGTTTA-3' (reverse); and osteopontin, 5'-TGCACCCAGATCCTA TAGCC-3' (forward) and 5'-CTCCATCGTCATCATCATCG-3' (reverse). The $20 \mu \mathrm{l}$ PCR system contained $2 \mu \mathrm{l}$ genomic DNA, $2 \mu \mathrm{l}$ dNTPs, $10 \mu 1$ buffer, $0.5 \mu 1$ Rox reference dye and 5 pmol of each primer. Thermal cycling was performed as follows: Denaturing at $95^{\circ} \mathrm{C}$ for $3 \mathrm{~min}$, then 30 cycles of $95^{\circ} \mathrm{C}$ for $30 \mathrm{sec}, 55^{\circ} \mathrm{C}$ for $30 \mathrm{sec}$ and $72^{\circ} \mathrm{C}$ for $30 \mathrm{sec}$, with elongation at $72^{\circ} \mathrm{C}$ for 10 min using a CFX96 Real-Time $\mathrm{C} 1000$ thermocycler (Bio-Rad Laboratories, Inc.). PCR products were electrophoresed on 1\% agarose gel (800669; Schwarz/Mann Biotech, Cleveland, OH, USA) at $200 \mathrm{~mA}$ until separation was achieved. DNA fragments were visualized using a long wave UV light box and images were captured using a Universal Hood II Gel Doc $^{\text {TM }}$ XR camera (1708170) and were analyzed using Image Lab software (both Bio-Rad Laboratories, Ltd.).

Statistical analysis. SPSS version 11.5 (SPSS, Inc., Chicago, IL, USA) was used for statistical analyses. Values are expressed as the mean \pm standard error of the mean. One-way analysis of variance was used to assess statistical significance. Survival curves were compared using the log-rank test. $\mathrm{P}<0.05$ was considered to indicate a statistically significant difference.

\section{Results}

Oxaliplatin induces accumulation of CAFs in the tumor microenvironment. Chemotherapy is widely used for the treatment of colorectal cancer; however, drug resistance has become problematic due to its increasing frequency. Several mechanisms of drug resistance of cancers have been proposed $(31,32)$; however, a consensus has not yet been reached and further elucidation is required. The present study hypothesized that CAFs may have an important role in drug resistance of cancers. 

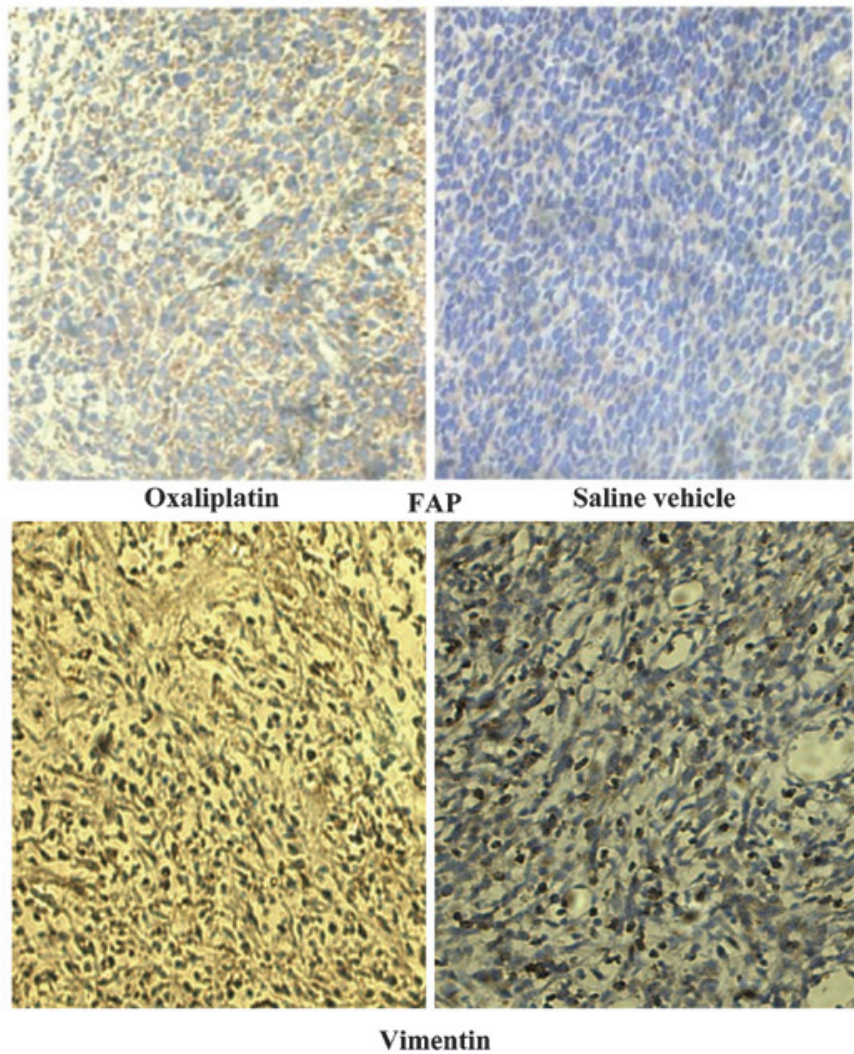

Figure 2. Effects of oxaliplatin on the accumulation of CAFs in CT26 colon cancer cell-derived murine xenograft tumors. Immunohistochemical analysis of tumors harvested on day 22 for FAP and an vimentin, which are specifically expressed in CAFs (magnification, x100 for FAP and x200 for vimentin). CAF, cancer-associated fibroblast; FAP, fibroblast activation protein.

To test this hypothesis, Balb/C mice ( $\mathrm{n}=5$ per group) were inoculated with CT26 colon carcinoma cells (day 0) and then treated with the saline vehicle or oxaliplatin for 14 days (from days 8-22). Subsequently, the mice were sacrificed via cervical dislocation and the tumor xenografts were excised, sectioned and stained using an anti-FAP and anti-vimentin antibodies. As shown in Fig. 2, treatment with oxaliplatin markedly increased the amount of FAP and vimentin, which are specific markers of CAFs, expressed in the stroma of the tumor tissues, indicating that oxaliplatin increased the accumulation of CAFs in the xenograft tumors.

Combined treatment with oxaliplatin and a pharmacological inhibitor of CAFs reduces tumor growth in vivo. In order to evaluate whether the ability of oxaliplatin to increase the accumulation of CAFs in the xenograft tumors was clinically relevant, the present study combined pharmacological inhibition of CAFs by PT-100 with the chemotherapeutic drug oxaliplatin, to which CT26 cells are partially sensitive, in a xenograft model. Mice ( $n=5$ per group) were subcutaneously injected with CT26 cells on day 0 and then treated with saline vehicle, PT-100 alone, oxaliplatin alone, or PT-100 combined with oxaliplatin from days 8-32. As shown in Fig. 3A, PT-100 combined with oxaliplatin suppressed tumor growth more significantly than treatment with either oxaliplatin or PT-100 alone $(\mathrm{P}<0.05)$. PT-100 combined with oxaliplatin also significantly increased the survival of the mice compared to that in the other three treatment groups, particularly the mice treated with saline vehicle, which died after 45-60 days (Fig. 3B).

Consistent with the results shown in Fig. 2, FAP, a specific marker for CAFs, was most highly expressed in the stroma of the tumors of the mice treated with oxaliplatin alone, indicating that oxaliplatin increased the accumulation of CAFs in the xenograft tumors. However, PT-100 combined with oxaliplatin markedly reduced the expression of FAP and therefore prevented the accumulation of CAFs in the stroma of the tumor tissues. In addition, TUNEL staining was performed on the xenograft tumor sections, which revealed that PT-100 combined with oxaliplatin increased the number of apoptotic tumor cells compared with that in the three other treatment groups (Fig. 3C-F).

Next, to investigate how oxaliplatin induced the accumulation of CAFs, RT-qPCR analysis was performed to assess the expression of a number of cytokines which are associated with the accumulation of CAFs in the xenograft tumor tissues. CAFs are hypothesized to accumulate in the tumor microenvironment by the following major mechanisms: Resident fibroblasts transforming into CAFs, the EMT and the EndMT $(8,10,11)$; these processes are mediated by several common cytokines, including TGF- $\beta 3$, FGF- 2 and osteopontin $(9,12)$. Thus, the present study quantitatively measured the abundance these cytokines in the xenograft tumor tissues. As shown in Fig. 3G, oxaliplatin increased the expression of $T G F-\beta 3$ and $F G F-2$ (basic FGF) by 1.79- and 2.63-fold, respectively, compared to those in the tumors of the saline vehicle-treated animals. Furthermore, the expression of $T G F-\beta 3$ and $F G F-2$ in the tumors of animals treated with PT-100 alone or with PT-100 combined with oxaliplatin was lower than that in the saline vehicle-treated animals. Although oxaliplatin treatment did not significantly increase the expression of osteopontin mRNA, its expression was significantly decreased in the tumors of the animals treated with PT-100 only or with PT-100 combined with oxaliplatin (0.28- and 0.16-fold, respectively, of that in the saline vehicle-treated group). These results indicated that pharmacological inhibition of CAFs using PT-100 reduced the tumor expression levels of cytokines known to promote the accumulation of CAFs.

Combed treatment with oxaliplatin and a pharmacological inhibitor of CAFs reduces the recruitment of tumor-associated macrophages (TAMs) and dendritic cells. The tumor microenvironment can affect the malignant potential of the tumor; tumor-associated macrophages and dendritic cells have an important role in this process $(33,34)$. Macrophages constitute an extremely heterogeneous population, including M2 (or alternatively activated) macrophages, which are now generally accepted to be TAMs. TAMs mostly exert pro-tumor functions by promoting tumor-cell survival, proliferation and dissemination. Tumor-associated dendritic cells (DCs) are another immune-regulatory cell population. A variety of sub-populations of tumor-associated DCs are known, among which CD11c+ DCs are able to promote tumorigenesis (35). In order to test whether CAFs interact with M2 macrophages or immature dendritic cells (imDCs) and tumor-infiltrating DCs (TIDCs), tumor cells from mice at day 22 were analyzed by flow cytometry. As shown in Fig. 4, the number of M2 macrophages (F4/80+) was highest in the saline vehicle-treated group and lowest in the xenograft tumors of animals treated with PT-100 combined with oxaliplatin; furthermore, tumors of animals 

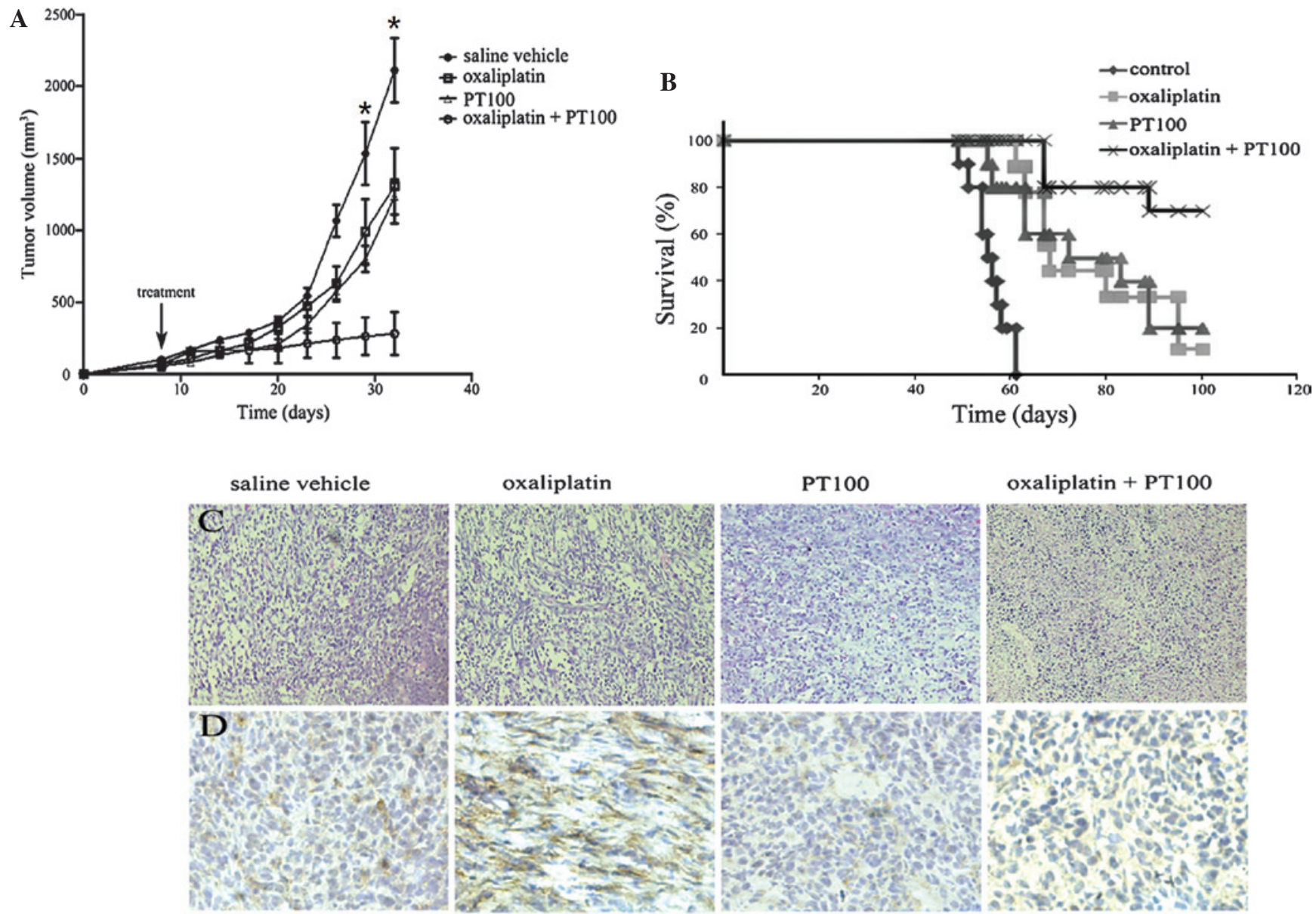

oxaliplatin

PT100

oxaliplatin + PT100
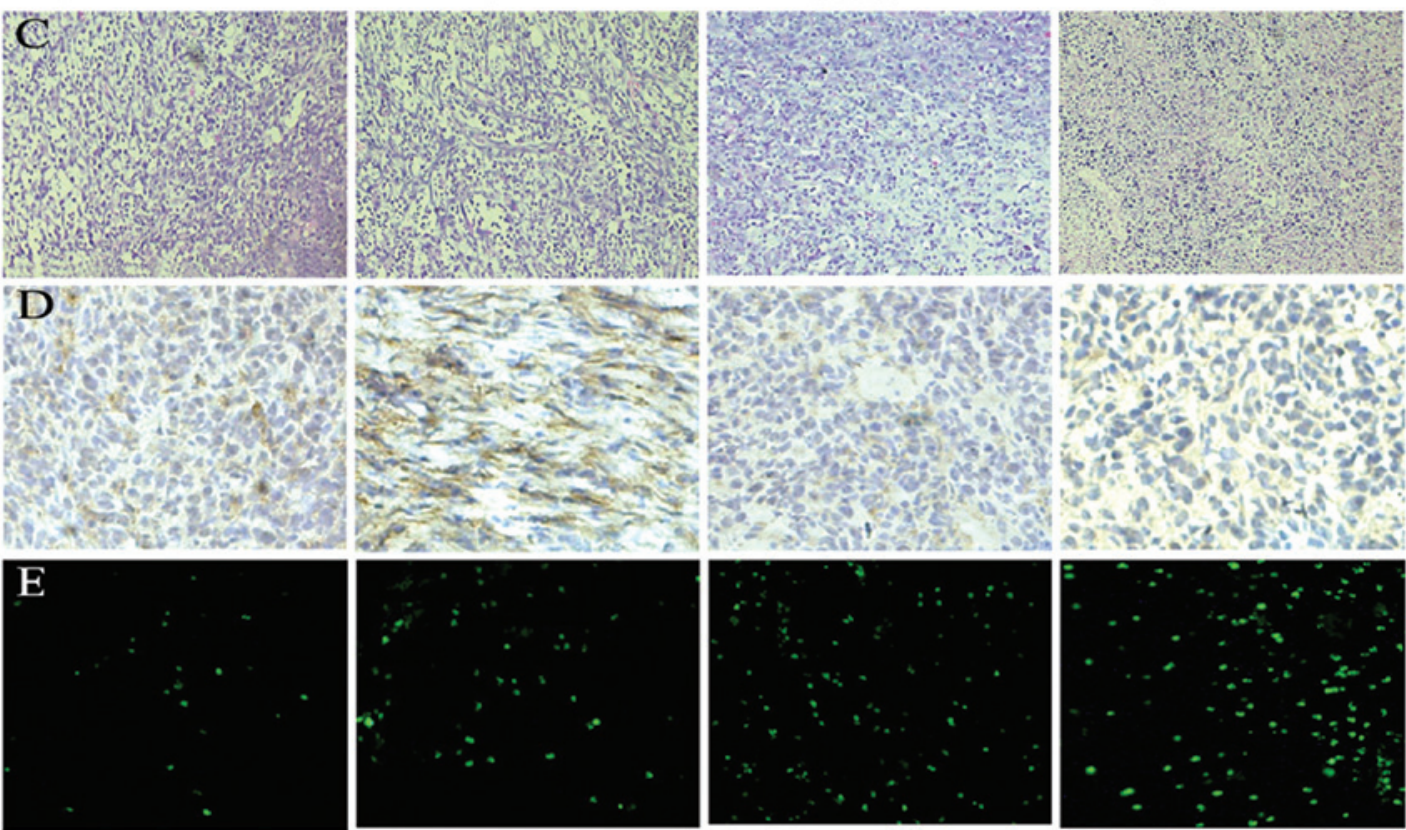

F

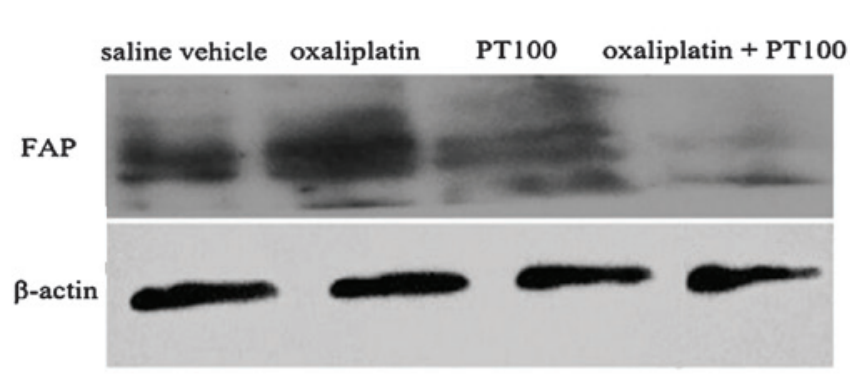

G
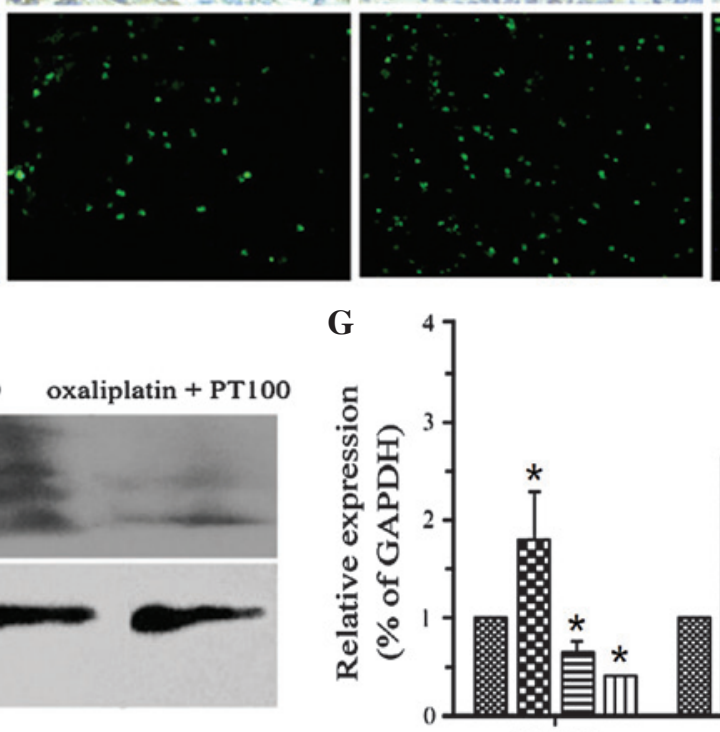

TGF- $\beta 3$
Saline vehicle Oxaliplatin 曷 PT100 Oxaliplatin+PT100

Figure 3. Effects of oxaliplatin and PT-100 combination therapy on the accumulation of CAFs in CT26 colon cancer cell-derived murine xenograft tumors. (A) Tumor growth curves. Values are expressed as the mean \pm standard error of the mean. * P $<0.05$ vs. the three treatment groups. (B) Survival curves. (C) Hematoxylin and eosin staining of tumors harvested on day 22 (magnification, x100). (D) Immunostaining for FAP, which is specifically expressed in CAFs, in tumors harvested on day 22 (magnification, $\mathrm{x} 200$ ). (E) Detection of apoptotic cells in tumors excised on day 22 using the Deadend ${ }^{\mathrm{TM}}$ fluorometric terminal deoxynucleotidyl transferase dUTP nick end labeling system. (F) Western blot of FAP in the lysates of tumors excised on day 22. (G) Effects of oxaliplatin and PT-100 combination therapy on the expression of cytokines associated with CAFs in CT26 colon cancer cell-derived murine xenograft tumors. Tumors were resected at day 22 and the cytokine expression levels were assessed using reverse-transcription quantitative polymerase chain reaction analysis. ${ }^{*} \mathrm{P}<0.05$ vs. the saline vehicle control. Values are expressed as the mean \pm standard error of the mean. CAF, cancer-associated fibroblast; FAP, fibroblast activation protein.

treated with PT-100 combined with oxaliplatin contained significantly lower amounts of M2 macrophages compared with those in the groups treated with saline vehicle, oxaliplatin alone and PT-100 alone $(\mathrm{P}<0.01)$. In addition, the number of imDCs and TIDCs was highest in the saline vehicle-treated tumors, while the tumors of animals treated with PT-100 
A

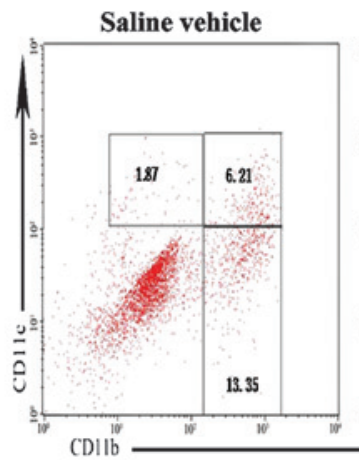

B

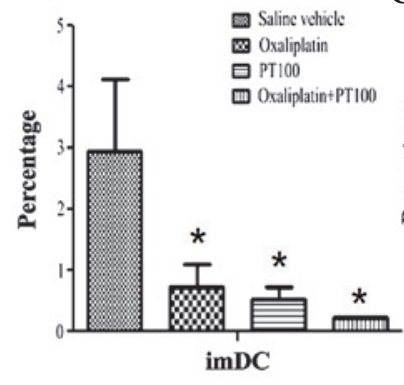

D
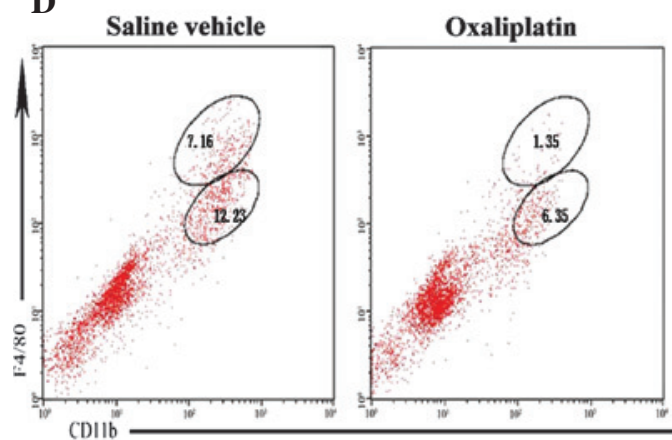

Oxaliplatin

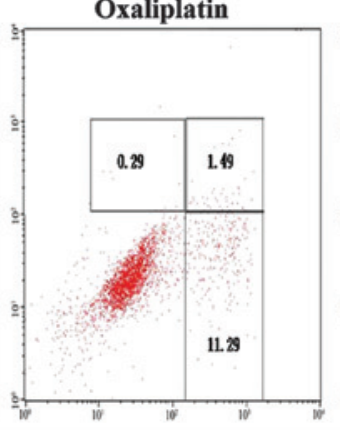

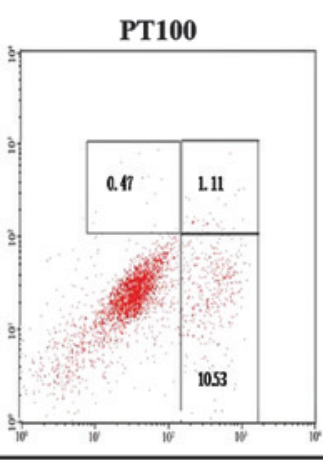

C

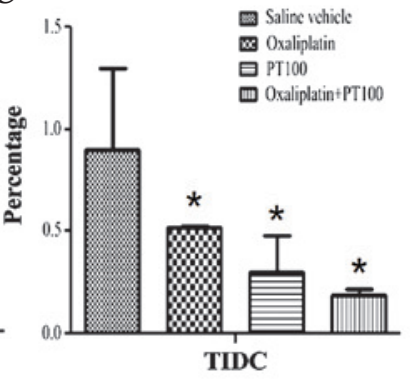

Oxaliplatin+PT100

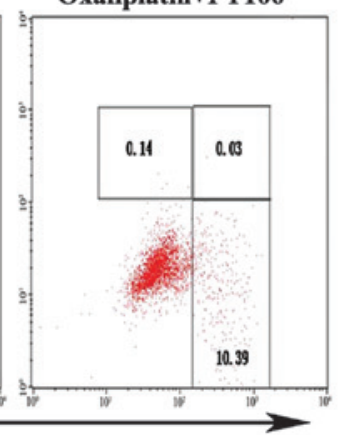

TIDC

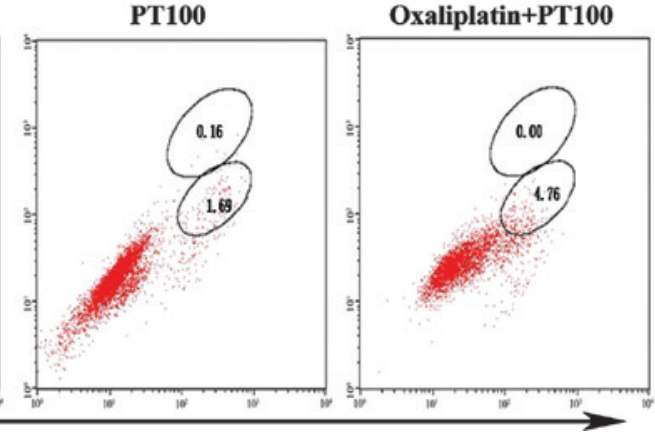

E

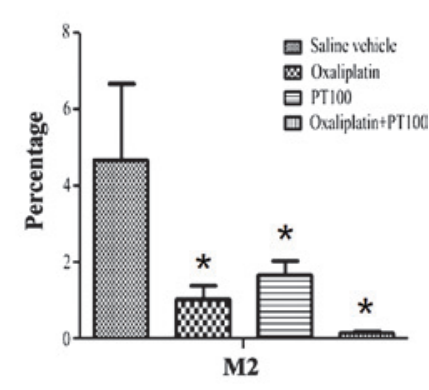

Figure 4. Effects of oxaliplatin and PT-100 combination therapy on the number of pro-tumorigenic immune cells in CT26 colon cancer cell-derived murine xenograft tumors. (A-C) Analysis of CD11b+ CD11c+tumor-associated dendritic cells. (D and E) Analysis of CD11b+ F4/80+ tumor-associated macrophages.

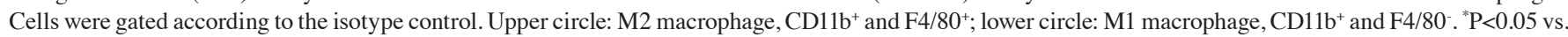
the saline vehicle control. Values are expressed as the mean \pm standard error of the mean. imDC, immature dendritic cells; TIDC, tumor-infiltrating dendritic cells.

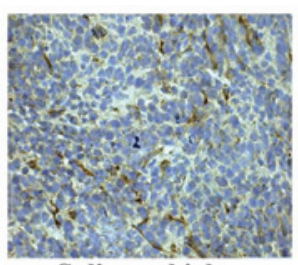

Saline vehicle

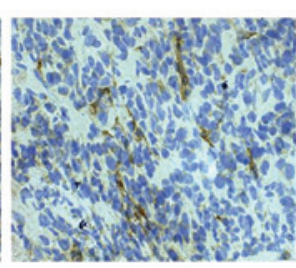

Oxaliplatin

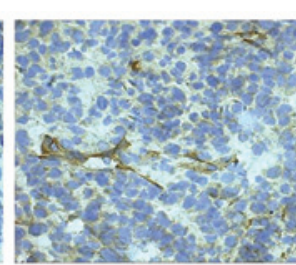

PT100

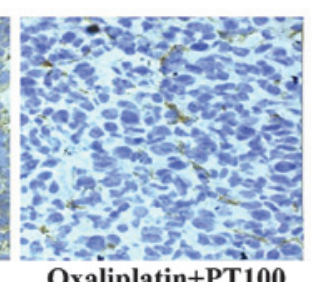

Oxaliplatin+PT100

Figure 5. Effects of oxaliplatin and PT-100 combination therapy on tumor angiogenesis in CT26 colon cancer cell-derived murine xenograft tumors. The tumor tissues were resected at day 22 and the density of blood vessels was indicated by immunostaining with an anti-CD31 antibody (magnification, x400).

combined with oxaliplatin contained a significantly lower number of imDCs and TIDCs, as compared with the groups treated with saline vehicle, oxaliplatin only and PT-100 only $(\mathrm{P}<0.05)$. These results indicated that pharmacological inhibition of CAFs using PT-100 in combination with oxaliplatin significantly reduced the recruitment of M2 macrophages and CD11c+ DCs to the xenograft tumors.

Combined treatment of oxaliplatin with a pharmacological inhibitor of CAFs reduces tumor angiogenesis. Angiogenesis, a key event required for tumor progression, is dependent on ECM remodeling (1). As an important source of ECM components, CAFs are able to promote angiogenesis during tumor growth and metastasis $(1,5)$. In order to test whether pharmacological inhibition of CAFs had any effect on angiogenesis in the tumor xenografts, immunohistochemical analysis was used to determine the density of CD31+ endothelial cells in the xenograft tumors at day 22. The tumors treated with PT-100 combined with oxaliplatin had a markedly lower density of CD31+ cells compared to that in the 
groups treated with saline vehicle, oxaliplatin alone and PT-100 alone (Fig. 5). These results indicated that pharmacological inhibition of CAFs using PT-100 in combination with oxaliplatin significantly reduced tumor angiogenesis.

\section{Discussion}

Chemotherapy has been a basic, vital and widespread means of treating colorectal cancer for numerous years; however, chemoresistance has become a significant obstacle. A number of studies have investigated the mechanisms of drug resistance to chemotherapeutic agents; it is mainly attributed to gene mutations, gene amplification, epigenetic changes that influence the uptake, metabolism or export of drugs from single cells $(36,37)$, or alterations to the signaling pathways which protect tumor stem cells (31). The tumor microenvironment has become a research hot spot, as it may be involved in the resistance of solid tumors to chemotherapy (38).

The present study demonstrated that the chemotherapeutic drug oxaliplatin increased the accumulation of CAFs in colon cancer xenograft tumor tissues, which may, to a certain extent, be responsible for drug resistance. The mechanisms by which oxaliplatin increased the accumulation of CAFs may be due to the upregulation of cytokines associated with the accumulation of CAFs. Studies have demonstrated that chemotherapy can increase hypoxia in the tumor microenvironment, and that such hypoxic conditions can induce a molecular response that drives the activation of a key transcription factor, namely the hypoxia-inducible factor (HIF). HIF regulates a variety of genes encoding cytokines that influence the growth, progression and metastasis of tumors $(39,40)$. In the present study oxaliplatin may possibly have induced hypoxia in the tumors, which may explain for the upregulation of TGF- $\beta 3$, FGF-2 and osteopontin observed in the xenograft tumors. TGF- $\beta$ is a multifunctional cytokine that regulates tissue morphogenesis and differentiation by influencing cell proliferation, differentiation, apoptosis and ECM production $(8,11)$. FGF-2, a prototypical pro-angiogenic factor, regulates a variety of important intracellular signal-transduction pathways, which mediate a series of cellular and molecular changes associated with the EMT (9). Osteopontin is a soluble ECM protein with known effector functions in tumor growth, angiogenesis and metastasis (12). All of these cytokines have been associated with the accumulation of CAFs $(8,9)$. As a result of the increased accumulation of CAFs, the secretion of these cytokines is elevated and eventually accelerates tumor growth (10-12).

Although the importance of CAFs in tumor progression has been recognized (41), the understanding of the mechanisms by which CAFs influence tumorigenesis remains limited. One generally accepted hypothesis is that an increase in the accumulation of CAFs, a vital component of the structure of the tumor microenvironment, may increase fibrosis in the tumor microenvironment, which may reduce drug uptake by the tumor cells (30). However, the present study suggested that CAFs induce the recruitment of inflammatory immune cells, which may facilitate tumor growth and progression. Tumor-associated macrophages and dendritic cells have been reported to produce matrix remodeling enzymes, reactive oxygen species and other bioactive molecules that influence cancer-cell proliferation, angiogenesis, invasion and metastasis $(35,42)$. In the present study, pharmacological inhibition of CAFs markedly suppressed the recruitment of pro-tumorigenic immune cells in vivo, which indicated that CAFs are able to influence the vital signaling pathways associated with the recruitment of pro-tumorigenic immune cells. It has been reported that CAFs orchestrate signaling processes associated with tumor-promoting inflammation in incipient neoplasia via a NF-kB signaling-dependent mechanism (15), while CAFs also modulated the tumor immune microenvironment in a 4T1 murine breast cancer model (43). In addition, as mentioned above, hypoxic tissues, in which the accumulation of CAFs is enhanced, secrete cytokines that undermine normal anti-tumor surveillance by macrophages, turning the macrophages into accomplices and facilitators of invasion and angiogenesis (44). However, in contrast to previous studies on tumor-associated immune cells $(30,43)$, no significant differences between the number of $\mathrm{CD} 4+/ \mathrm{CD} 8+\mathrm{T}$ cells were identified among the four experimental groups of the present study. Therefore, the role of CAFs as tumor immune modulators in tumorigenesis requires further study.

Apart from recruiting inflammatory immune cells, the pro-tumor function of CAFs may also be associated with the induction of angiogenesis within the tumor stroma. Angiogenesis, the formation of new blood vessels, is required for malignant tumor growth and metastasis (45). The findings of the present study demonstrated that pharmacological inhibition of CAFs simultaneously suppressed angiogenesis in the tumor xenografts. CAFs may affect angiogenesis at several levels. CAFs secret growth factors, includings VEGF and FGF-2, which are known to be important for endothelial cell migration (13). Moreover, CAFs are capable of remodeling connective tissue, and interact with epithelial cells and other cell types in connective tissues, which may promote angiogenesis (46). In addition, inflammatory immune cells may also affect tumor angiogenesis $(15,42)$. The present study demonstrated that pharmacological inhibition of CAFs reduced the recruitment of inflammatory immune cells, which indicated that CAFs may promote tumor angiogenesis by recruiting inflammatory immune cells. It has recently been reported that vascular endothelial cells and CAFs express similar genes (47). Thus, it is necessary to study the similarities and differences between CAFs and tumor endothelial cells in order to accurately guide the clinical application of anti-angiogenic agents.

In conclusion, the present study demonstrated that chemotherapy with oxaliplatin increased the accumulation of CAFs in the stroma of colon tumors in vivo, whereas pharmacological inhibition of CAFs alongside oxaliplatin treatment reduced the accumulation of CAFs, enhanced the response to oxaliplatin chemotherapy as well as reduced the expression of cytokines associated with the accumulation of CAFs, the recruitment of pro-tumorigenic immune cells and angiogenesis. Chemotherapy combined with treatments which inhibit the recruitment, formation or activity of CAFs may represent a novel method of improving the tumor response to chemotherapy and help to overcome resistance to chemotherapeutic agents.

\section{Acknowledgements}

The present study was supported by grants from the National Science Foundation of China (grant nos. 81123003 and 81201787). 


\section{References}

1. Kessenbrock K, Plaks V and Werb Z: Matrix metalloproteinases: Regulators of the tumor microenvironment. Cell 141: 52-67, 2010.

2. Ozao-Choy J, Ma G, Kao J, Wang GX, Meseck M, Sung M, Schwartz M, Divino CM, Pan PY and Chen SH: The novel role of tyrosine kinase inhibitor in the reversal of immune suppression and modulation of tumor microenvironment for immune-based cancer therapies. Cancer Res 69: 2514-2522, 2009.

3. Swartz MA, Lida N, Roberts EW, Sangaletti S, Wong MH, Yull FE, Coussens LM and DeClerck YA: Tumor microenvironment complexity: Emerging roles in cancer therapy. Cancer Res 72: 2473-2480, 2012

4. Albini A and Sporn MB: The tumour microenvironment as a target for chemoprevention. Nat Rev Cancer 7: 139-147, 2007.

5. Zi F, He J, He D, Li Y, Yang L and Cai Z: Fibroblast activation protein $\alpha$ in tumor microenvironment: recent progression and implications (review). Mol Med Rep 11: 3203-3211, 2015.

6. Whiteside TL: The tumor microenvironment and its role in promoting tumor growth. Oncogene 27: 5904-5912, 2008.

7. Mueller MM and Fusenig NE: Friends or foes - bipolar effects of the tumour stroma in cancer. Nat Rev Cancer 4: 839-849, 2004

8. Kojima Y, Acar A, Eaton EN, Mellody KT, Scheel C, Ben-Porath I, Onder TT, Wang ZC, Richardson AL, Weinberg RA and Orimo A: Autocrine TGF-beta and stromal cell-derived factor-1(SDF-1) signaling drives the evolution of tumor-promoting mammary stromal myofibroblasts. Proc Natl Acad Sci USA 107: 20009-20014, 2010.

9. Billottet C, Tuefferd M, Gentien D, Rapinat A, Thiery JP, Broët $\mathrm{P}$ and Jouanneau J: Modulation of several waves of gene expression during FGF-1 induced epithelial-mesenchymal transition of carcinoma cells. J Cell Biochem 104: 826-839, 2008.

10. Potenta S, Zeisberg E and Kalluri R: The role of endothelial-to-mesenchymal transition in cancer progression. Br J Cancer 99: 1375-1379, 2008

11. Xu J, Lamouille S and Derynck R: TGF-beta-induced epithelial to mesenchymal transition. Cell Res 19: 156-172, 2009.

12. Anderberg C, Li H, Fredriksson L, Andrae J, Betsholtz C, Li $\mathrm{X}$, Eriksson $\mathrm{U}$ and Pietras $\mathrm{K}$ : Paracrine signaling by platelet-derived growth factor-CC promotes tumor growth by recruitment of cancer-associated fibroblasts. Cancer Res 69 : 369-378, 2009.

13. Hughes CC: Endothelial-stromal interactions in angiogenesis Curr Opin Hematol 15: 204-209, 2008.

14. Yang L, Pang Y and Moses HL: TGF-beta and immune cells: An important regulatory axis in the tumor microenvironment and progression. Trends Immunol 31: 220-227, 2010.

15. Erez N, Truitt M, Olson P, Arron ST and Hanahan D: Cancer-Associated Fibroblasts Are Activated in Incipient Neoplasia to Orchestrate Tumor-Promoting Inflammation in an NF-kappaB-Dependent Manner. Cancer Cell 17: 135-147, 2010.

16. Fukumura D, Xavier R, Sugiura T, Chen Y, Park EC, Lu N, Selig M, Nielsen G, Taksir T, Jain RK and Seed B: Tumor induction of VEGF promoter activity in stromal cells. Cell 94: 715-725, 1998

17. Infante JR, Matsubayashi H, Sato N, Tonascia J, Klein AP, Riall TA, Yeo C, Iacobuzio-Donahue C and Goggins M: Peritumoral fibroblast SPARC expression and patient outcome with resectable pancreatic adenocarcinoma. J Clin Oncol 25: 319-325, 2007.

18. Micke P, Kappert K, Ohshima M, Sundquist C, Scheidl S, Lindahl P, Heldin $\mathrm{CH}$, Botling J, Ponten F and Ostman A: In situ identification of genes regulated specifically in fibroblasts of human basal cell carcinoma. J Invest Dermatol 127 $1516-1523,2007$.

19. Tsujino T, Seshimo I, Yamamoto H, Ngan CY, Ezumi K, Takemasa I, Ikeda M, Sekimoto M, Matsuura N and Monden M: Stromal myofibroblasts predict disease recurrence for colorectal cancer. Clin Cancer Res 13: 2082-2090, 2007.

20. Aertgeerts K, Levin I, Shi L, Snell GP, Jennings A, Prasad GS, Zhang Y, Kraus ML, Salakian S, Sridhar V, et al: Structural and kinetic analysis of the substrate specificity of human fibroblast activation protein alpha. J Biol Chem 280: 19441-19444, 2005.

21. Aggarwal S, Brennen WN, Kole TP, Schneider E, Topaloglu O, Yates M, Cotter RJ and Denmeade SR: Fibroblast activation protein peptide substrates identified from human collagen I derived gelatin cleavage sites. Biochemistry 47: 1076-1086, 2008.
22. Edosada CY, Quan C, Wiesmann C, Tran T, Sutherlin D, Reynolds M, Elliott JM, Raab H, Fairbrother W and Wolf BB: Selective inhibition of fibroblast activation protein protease based on dipeptide substrate specificity. J Biol Chem 281: 7437-7444, 2006

23. Ostermann E, Garin-Chesa P, Heider KH, Kalat M, Lamche H, Puri C, Kerjaschki D, Rettig WJ and Adolf GR: Effective immunoconjugate therapy in cancer models targeting a serine protease of tumor fibroblasts. Clin Cancer Res 14: 4584-4592, 2008.

24. Niedermeyer J, Enenkel B, Park JE, Lenter M, Rettig WJ, Damm K and Schnapp A: Mouse fibroblast-activation protein: Conserved Fap gene organization and biochemical function as a serine protease. Eur J Biochem 254: 650-654, 1998.

25. Cheng JD, Dunbrack RL Jr, Valianou M, Rogatko A, Alpaugh RK and Weiner LM: Promotion of tumor growth by murine fibroblast activation protein, a serine protease in an animal model. Cancer Res 62: 4767-4772, 2002.

26. Rasmussen HB, Branner S, Wiberg FC and Wagtmann N: Crystal structure of human dipeptidyl peptidase IV/CD26 in complex with a substrate analog. Nat Struct Biol 10: 19-25, 2003.

27. Jones B, Adams S, Miller GT, Jesson MI, Watanabe T and Wallner BP: Hematopoietic stimulation by a dipeptidyl peptidase inhibitor reveal a novel regulatory mechanism and therapeutic treatment for blood cell deficiencies. Blood 102: 1641-1648, 2003

28. Diaz-Rubio E, Sastre J,Zaniboni A, Labianca R, Cortés-Funes H, de Braud F, Boni C, Benavides M, Dallavalle G and Homerin M: Oxaliplatin as single agent in previously untreated colorectal carcinoma patients: A phase II multicentric study. Ann Oncol 9: 105-108, 1998.

29. Raymond E, Chaney SG, Taamma A and Cvitkovic E: Oxaliplatin: A review of preclinical and clinical studies. Ann Oncol 9: 1053-1071, 1998.

30. Loeffler M, Krüger JA, Niethammer AG and Reisfeld RA: Targeting tumor-associated fibroblasts improves cancer chemotherapy by increasing intratumoral drug uptake. J Clin Invest 116: 1955-1962, 2006.

31. Dean M: ABC transporters, drug resistance, and cancer stem cells. J Mammary Gland Biol Neoplasia 14: 3-9, 2009.

32. Redmond KM, Wilson TR, Johnston PG and Longley DB: Resistance mechanisms to cancer chemotherapy. Front Biosci 13: 5138-5154, 2008.

33. Ma Y, Shurin GV, Gutkin DW and Shurin MR: Tumor associated regulatory dendritic cells. Semin Cancer Biol 22: 298-306, 2012.

34. Qian BZ and Pollard JW: Macrophage diversity enhances tumor progression and metastasis. Cell 141: 39-51, 2010.

35. Cubillos-Ruiz JR, Engle X, Scarlett UK, Martinez D, Barber A, Elgueta R, Wang L, Nesbeth Y, Durant Y and Gewirtz AT: Polyethylenimine-based siRNA nanocomplexes reprogram tumor-associated dendritic cells via TLR5 to elicit therapeutic antitumor immunity. J Clin Invest 119: 2231-2244, 2009.

36. Perugorria MJ, Castillo J, Latasa MU, Goñi S, Segura V, Sangro B, Prieto J, Avila MA and Berasain C: Wilms' tumor 1 gene expression in hepatocellular carcinoma promotes cell dedifferentiation and resistance to chemotherapy. Cancer Res 69: 1358-1367, 2009.

37. Plumb JA, Strathdee G, Sludden J, Kaye SB and Brown R: Reversal of drug resistance in human tumor xenografts by 2'-deoxy-5-azacytidine-induced demethylation of the hMLH1 gene promoter. Cancer Res 60: 6039-6044, 2000.

38. Trédan O, Galmarini CM, Patel K and Tannock IF: Drug resistance and the solid tumor microenvironment. J Natl Cancer Inst 99: 1441-1454, 2007.

39. Brahimi-Horn MC, Chiche J and Pouysségur J: Hypoxia and cancer. J Mol Med (Berl) 85: 1301-1307, 2007.

40. Pouysségur J, Dayan F and Mazure NM: Hypoxia signalling in cancer and approaches to enforce tumour regression. Nature 441: 437-443, 2006.

41. Kalluri R and Zeisberg M: Fibroblasts in cancer. Nat Rev Cancer 6: 392-401, 2006

42. Condeelis J and Pollard JW: Macrophages: Obligate partners for tumor cell migration, invasion and metastasis. Cell 124: 263-266, 2006.

43. Liao D, Luo Y, Markowitz D, Xiang R and Reisfeld RA: Cancer associated fibroblasts promote tumor growth and metastasis by modulating the tumor immune microenvironment in a 4T1 murine breast cancer mode. PLoS One 4: e7965, 2009. 
44. DeClerck K and Elble RC: The role of hypoxia and acidosis in promoting metastasis and resistance to chemotherapy. Front Biosci (Landmark Ed) 15: 213-225, 2010.

45. Saharinen P, Eklund L, Pulkki K, Bono P and Alitalo K: VEGF and angiopoietin signaling in tumor angiogenesis and metastasis. Trends Mol Med 17: 347-362, 2011.
46. Desmoulière $A$, Guyot $\mathrm{C}$ and Gabbiani G: The stroma reaction myofibroblast: A key player in the control of tumor cell behavior. Int J Dev Biol 48: 509-517, 2004

47. Zeisberg EM, Potenta S, Xie L, Zeisberg M and Kalluri R: Discovery of endothelial to mesenchymal transition as a source for carcinoma-associated fibroblasts. Cancer Res 67: 10123-10128, 2007. 